\title{
Balkanologie
}

Balkanologie Revue d'études pluridisciplinaires

Vol. IX, $n^{\circ}$ 1-2 | 2005

Volume IX Numéro 1-2

\section{Les représentations orthodoxes auprès de l'Union européenne : entre concurrence inter-orthodoxe et dynamiques d'européanisation}

Orthodox offices towards the European Union: inter-orthodox competition and dynamics of europeanization

\section{Bérengère Massignon}

\section{(2) OpenEdition Journals}

\section{Édition électronique}

URL : http://journals.openedition.org/balkanologie/599

DOI : 10.4000/balkanologie.599

ISSN : 1965-0582

\section{Éditeur}

Association française d'études sur les Balkans (Afebalk)

Édition imprimée

Date de publication : 1 décembre 2005

ISSN : 1279-7952

\section{Référence électronique}

Bérengère Massignon, «Les représentations orthodoxes auprès de l'Union européenne : entre concurrence inter-orthodoxe et dynamiques d'européanisation », Balkanologie [En ligne], Vol. IX, n 1-2 I 2005, mis en ligne le 13 janvier 2010, consulté le 17 décembre 2020. URL : http://

journals.openedition.org/balkanologie/599; DOI : https://doi.org/10.4000/balkanologie.599 


\title{
LES REPRÉSENTATIONS ORTHODOXES AUPRÈS DE L'UNION EUROPÉENNE : ENTRE CONCURRENCE INTER-ORTHODOXE ET DYNAMIQUES D'EUROPÉANISATION
}

\author{
Bérengère Massignon*
}

L'inscription européenne de l'orthodoxie présente des difficultés. Elle est marquée par un paradoxe. Branche presque exclusivement européenne du christianisme, à la différence du catholicisme et du protestantisme majoritairement implantés hors d'Europe, l'orthodoxie se situe pourtant en marge de l'Europe, comme territoire mais aussi comme civilisation occidentale, définie par les ruptures de la Réforme, des Lumières et de la Révolution française. L'orthodoxie resta à l'écart des grands courants de modernisation politiques, économiques, sociaux, scientifiques, intellectuels et religieux qui ont fait le socle de la spécificité civilisationnelle européenne. Ceci se traduit par un sentiment d'extériorité à l'Europe, a fortiori vis-à-vis de l'Union européenne (UE), voire même, dans certains cas, d'hostilité. En effet, l'orthodoxie a parfois été le vecteur d'un sentiment anti-occidental, combiné à une critique et même un refus de la modernité ${ }^{\text {. }}$.

De plus, l'orthodoxie a une situation minoritaire dans l'UE ; seuls deux pays à majorité orthodoxe en sont actuellement membres, à savoir la Grèce depuis 1980, et Chypre depuis le $1^{\mathrm{er}}$ mai 2004. Ce statut contraste avec le poids de l'orthodoxie dans des pays où la définition de l'identité nationale se confond avec l'appartenance à la religion orthodoxe. Les mobilisations grecques, avec, en tête, l'Église orthodoxe, contre la jurisprudence de la Cour européenne des droits de l'Homme et les pressions de l'UE qui exigeaient la suppression de la

\footnotetext{
"Membre du Groupe "Sociétés, Religions et Laïcités " (UMR CNRS-EPHE). Chargée de cours à Sciences Po Paris. E-mail : 〈bemassignon@hotmail.com〉
}

\footnotetext{
${ }^{1}$ Makrides (Vasilios N.), " Le rôle de l'orthodoxie dans la formation de l'anti-européanisme et de l'antioccidentalisme grecs ", in Vincent (Gilbert), Willaime (Jean-Paul), éds., Religions et transformations de l'Europe, Strasbourg : Presses Universitaires de Strasbourg, 1993.
} 
mention de la religion sur les cartes d'identité ${ }^{2}$, considérée comme potentiellement discriminatoire pour les minorités religieuses, illustrent les frustrations suscitées par ce décalage entre le statut majoritaire sur le plan interne et la situation minoritaire au sein de l'UE.

Les Églises orthodoxes sont néanmoins amenées à s'adapter à la nouvelle donne géopolitique ouverte par la chute du mur de Berlin et l'implosion de l'Empire soviétique. L'émancipation des pays frères $a$, notamment, ouvert des perspectives nouvelles d'élargissement de l'Europe communautaire à l'Est. Le ${ }_{1}{ }^{\mathrm{er}}$ mai 2004, dix nouveaux entrants ont rejoint les Quinze. Ces nouveaux membres sont majoritairement issus de l'ancienne sphère d'influence soviétique. Certains comptent des minorités orthodoxes. En Finlande, membre de l'UE depuis 1995, l'orthodoxie est religion d'État aux côtés de l'Église luthérienne ${ }^{3}$. Les orthodoxes sont également présents dans les Pays baltes 4 , intégrés à l'UE en 2004. Les prochaines vagues d'élargissement concernent des pays à majorité orthodoxe. La Roumanie et la Bulgarie pourraient rejoindre l'Union européenne en 2007 , suivies à terme par les pays des Balkans et peut-être l'Ukraine. Ces adhésions ou candidatures à l'adhésion rapprochent le territoire de l'Union de la Russie, débouchant sur de nouvelles interactions. L'implication de la Pologne dans la Révolution Orange en Ukraine en a fourni un témoignage récent. Le démantèlement de l'Union soviétique, ainsi que de la Yougoslavie, a bouleversé, en outre, le paysage canonique de l'orthodoxie car il a conduit à la création de nouvelles Églises orthodoxes autocéphales. De plus, la chute du communisme a induit en Russie un retour du sentiment religieux, vecteur de

\footnotetext{
${ }^{2}$ Molokotos-Liederman (Lina), "Identity Crisis: Greece, Orthodoxy and the European Union ", Journal of Contemporary Religion, 18 (3), 2003.

${ }^{3}$ Malgré ce statut égal, les orthodoxes ne représentent que $1 \%$ de la population contre $83,6 \%$ de luthériens. Source : US International Report on Religious Freedom, 2005.

4 Il n'est pas facile d'évaluer la population orthodoxe des Pays baltes. En l'absence de statistiques fiables, le gouvernement américain donne des chiffres basés sur des modes de comptage différents : soit les données fournies par les Églises aux gouvernements nationaux ; soit une déduction fondée sur des statistiques ethniques car les orthodoxes sont généralement des russophones, non citoyens et résidents permanents. En Lettonie, selon l'auto-déclaration des Églises, les trois principales confessions sont, dans l'ordre : les luthériens (539 000), les catholiques (428 000) et les orthodoxes (350 000). Rapporté au chiffre total de la population, on aurait $22,46 \%$ de luthériens, $17,83 \%$ de catholiques et $14,58 \%$ d'orthodoxes. La religion majoritaire en Estonie est le luthéranisme (170 000). Le cas de l'Estonie retient l'attention de Moscou car il existe deux Églises orthodoxes : l'une - l'Église orthodoxe apostolique d'Estonie (18 ooo fidèles) - est indépendante ; l'autre - l'Église orthodoxe d'Estonie (150 000 fidèles) - est rattachée au Patriarcat de Moscou. Si on additionne les membres des deux Églises orthodoxes, on en déduit que luthériens et orthodoxes font à peu près jeu égal et représentent, chacun, quelque $12,5 \%$ de la population. En Lituanie où la majorité est catholique, les orthodoxes forment le deuxième groupe confessionnel avec 140 ooo membres, soit 4,12\% de la population. Mais, si l'on s'appuie sur des statistiques ethniques, la population lituanienne, présumée catholique, forme $68 \%$ de la population globale et les russophones (Russes, Ukrainiens, et Biélorusses), présumés orthodoxes, 29 \%. Source : US International Report on Religious Freedom, 2005.
} 
nationalisme, alimenté, notamment, par un refus de l'ouverture du marché religieux aux missions protestantes ou catholiques 5 .

Ces bouleversements géopolitiques et religieux expliquent le désir de l'Église orthodoxe de Russie de se positionner sur le terrain du lobbying en direction des institutions européennes. Elle se trouve alors en concurrence avec les deux représentations existantes : celle du Patriarcat œcuménique de Constantinople et celle de l'Église orthodoxe de Grèce. Qui des "trois Rome" (Athènes, Constantinople ou Moscou) est habilité à représenter et défendre les intérêts orthodoxes en Europe occidentale, notamment ceux des diasporas orthodoxes, anciennement implantées à l'Ouest ou issues d'une émigration récente ?

Nous examinerons dans un premier temps la genèse de ces trois représentations orthodoxes auprès des institutions européennes, puis les clivages qui opposent Patriarcat œcuménique et Églises nationales dans leurs diverses visions de l'orthodoxie dans l'Europe.

La présence à Bruxelles nécessite une dynamique d'européanisation que nous définissons comme :

le processus de professionnalisation par lequel les organismes religieux et philosophiques présents à Bruxelles s'adaptent au jeu européen, notamment aux exigences du lobbying en direction des institutions europeennes et aux attentes de la Commission européenne à l'égard des religions et des mouvements humanistes. Nous faisons l'hypothèse que cette logique adaptative crée de la collaboration transnationale et trans-confessionnelle, élément de formation d'une société civile européenne $^{6}$.

Ainsi, nous montrerons la manière dont les Églises orthodoxes s'adaptent au jeu communautaire, en développant un processus de coopération inter-orthodoxe et œcuménique, tout en renforçant leurs moyens de lobbying en direction des institutions européennes. Dans leurs stratégies d'européanisation les Églises orthodoxes font néanmoins montre de spécificités, sans cesse réaménagées et négociées.

\footnotetext{
5 Sur le réemploi des ressources religieuses et la tendance au protectionnisme religieux dans la Russie post-soviétique, voir : Rousselet (Kathy), " L'Église orthodoxe russe dans l'espace soviétique et post-soviétique des années soixante à nos jours ", in Mayeur (Jean-Marie), Pietri (Charles et Luce), Vauchez (Andrè), Venard (Marc), èds., Histoire du christianisme, crise et renouveau (de 1958 à nos jours), Paris : Desclée, 2000 ; Rousselet (Kathy), "Globalisation et territoire religieux en Russie ", in Bastian, (JeanPierre), Champion, (Françoise), Rousselet, (Kathy), éds., La globalisation du religieux, Paris : L'Harmattan, 2001 ; Kalinowski (Wojtek), Moniak-Azzopardi (Agnieszka), " Réemploi du religieux dans la géopolitique. Le cas des identités collectives russes et européennes n, Études, février 2003.
}

\footnotetext{
${ }^{6}$ Massignon (Bérengère), " Les relations des organismes européens religieux et humanistes avec les institutions de l'Union européenne : logiques nationales et confessionnelles et dynamique d'européanisation ", Commissariat au Plan, Institut Universitaire de Florence, Chaire Jean Monnet d'Etudes européennes, Croyances religieuses, morales et éthiques dans le processus de construction européenne (Actes du colloque du Commissariat au Plan " Europe et religions "), Paris : La Documentation française, 2002, p. 23.
} 


\section{LA CONCURRENCE INTRA-ORTHODOXE À BRUXELLES}

À la différence des catholiques ou des protestants représentés au sein d'une organisation paneuropéenne unitaire (la COMECE pour les catholiques et l'EECCS, puis la KEK pour les protestants) ${ }^{7}$, l'orthodoxie ne dispose pas d'une seule instance auprès des institutions européennes. Trois bureaux se sont ouverts successivement, au rythme de la construction européenne et de l'élargissement de l'Union. À la diversité des structures correspondent aussi différentes manières de définir la place de l'orthodoxie par rapport à l'identité européenne.

\section{Trois représentations de l'orthodoxie auprès des institutions européennes}

Le lien entre orthodoxie et nation se traduit sur le plan ecclésiologique par l'existence d'Églises autocéphales ${ }^{8}$. Ceci induit l'éparpillement des représentations orthodoxes à Bruxelles. Il existe une concurrence entre, d'une part, le Patriarcat oecuménique qui a la primauté d'honneur, le rôle leader en matière de relations inter-orthodoxes ainsi, qu'en principe, l'autorité sur les diasporas orthodoxes en Occident et, d'autre part, les Églises orthodoxes nationales : celle de Grèce et, plus récemment, celle de Russie. Cette dernière, dans un communiqué publié dans Europaica ${ }^{9}$, dénie au Patriarcat de Constantinople le droit de parler au nom de toutes les Églises orthodoxes sans un accord préalable. Elle revendique aussi un accès direct, pour chaque Église, aux institutions européen-

7 La COMECE ou la Commission des Épiscopats de la Communauté européenne a été créée en 1980. L'EECCS ou Ecumenical Commission for Church and Society - une organisation strictement protestante et limitée à la seule Union européenne - a été fondėe dans les années soixante, puis a fusionné en 1999 avec la KEK ou Conférence des Églises européennes - une structure paneuropéenne et œcuménique, rassemblant, depuis 1959, anglicans, protestants et orthodoxes - , pour devenir la Commission "Église et Société " de la KEK (CES-CEC).

${ }^{8}$ Sur le lien orthodoxie/nation, voire notamment : Gillet (Olivier), Les Balkans. Religions et nationalisme, Bruxelles : Ouisa, 2001. Sur l'articulation complexe et différente des dimensions européenne et nationale pour chaque confession chrétienne voir : Bastian (Jean-Pierre), Collange, (Jean-François), éds., L'Europe à la recherche de son âme, Les Églises entre l'Europe et la nation, Colloque au Conseil de l'Europe du 4-5 novembre 1996, Genève : Labor et Fides, 1999. Pour une analyse traitant de ce même sujet à propos des trois confessions chrétiennes, mais aussi pour les juifs, les musulmans et les laïco-humanistes séculiers, voir Massignon (Bérengère), La construction européenne : un "laboratoire " pour la gestion de la pluralité religieuse. Clivages nationaux et confessionnels et dynamiques d'européanisation, EPHE, Thèse sous la direction de Jean-Paul Willaime, 2005, 2 Vol., notamment chapitre I, " Institutionnalisation et pluralisation de la scène religieuse européenne ".

9 Lettre du métropolite Kirill à Win Van Velzen, vice-président du Parti populaire européen, Europaica, (31), 19 janvier 2004. (Europaica est le journal bimensuel de l'Église orthodoxe russe, consacré à l'Europe, sur internet : 〈www.orthodoxeurope.org〉. Son premier numéro date du 26 novembre 2002). Or le Patriarcat a, en principe, le rôle leader dans les relations inter-orthodoxes. Sur les fonctions du Patriarcat œuménique, voir Papathomas (Grigorios D.), " Les différentes modalités d'exercice de la juridiction du Patriarcat de Constantinople ", Istina, 1995 ; Stavridis (Basile), " L'autoritè du patriarche œecuménique dans la vie de l'Église orthodoxe ", Istina, 1995. 
nes. L'existence de trois représentations traduit un désir d'indépendance de la part des Églises nationales, mais aussi une concurrence pour le leadership.

Le Patriarcat œcuménique ouvrit la première représentation orthodoxe auprès des institutions européennes. Le patriarche Bartholomé $1^{\mathrm{er}}$ s'engagea $^{\prime}$ personnellement en rendant visite à Jacques Delors, alors président de la Commission européenne (12 mai 1993). En avril 1994, lors d'un voyage à Strasbourg, il prononça un discours devant le Parlement européen et, le 10 janvier 1995, était inauguré un bureau à Bruxelles sous la dénomination révélatrice : " Bureau de l'Église orthodoxe auprès de l'UE ". Par ce nom, le Patriarcat œuménique indiquait sa volonté de représenter l'ensemble de l'orthodoxie auprès des institutions de l'Union. Ainsi, en septembre 1995, lors de la conférence des communautés locales orthodoxes à Istanbul, le patriarche Bartholomé $1^{\mathrm{er}}$ encouragea les liens entre les Églises orthodoxes et le bureau bruxellois, en vue de se mobiliser pour la Conférence intergouvernementale (CIG) préparant le Traité d'Amsterdam qui devait s'ouvrir en mars 1996.

Cet engagement tient à un double contexte. Avec l'effondrement du bloc de l'Est, un renouveau des relations inter-orthodoxes était espéré sous le leadership du Patriarcat œcuménique. Ensuite, sur le plan européen, le président Delors avait entamé, depuis 1990, une série de contacts avec des représentants protestants, puis catholiques. Il souhaitait les impliquer dans la définition d'un projet mobilisateur pour l'Europe d'après le Traité de Maastricht (1992). Selon Jacques Delors, 1992 représentait un tournant stratégique avec l'achèvement du grand marché unique. L'Europe devait désormais se constituer en communauté politique, ce qui supposait de préciser le socle commun de valeurs permettant de susciter l'adhésion des citoyens au projet européen. Delors avait coutume de dire : " on ne tombe pas amoureux d'un grand marché ". Ce désir fut affirmé dans son fameux discours de 1992 : " si dans les dix années à venir, nous n'arrivons pas à donner un sens, un souffle, une âme à l'Europe, nous aurons perdu la partie $»^{10}$. Pour la Commission, il était clair que le Patriarcat œcuménique était l'interlocuteur légitime orthodoxe. Désir auquel répondit l'engagement personnel du patriarche Bartholomé ainsi que l'ouverture rapide d'un bureau dans la capitale européenne.

Par contraste, l'institutionnalisation d'une représentation de l'Église de Grèce à Bruxelles fut beaucoup plus longue et réactive, suivant, plus qu'anticipant, les grandes étapes de la construction européenne. Peu après l'adhésion de la Grèce, le Saint Synode de Grèce décida, le 23 septembre 1980, la création

${ }^{10}$ Cette phrase, souvent citée, provient d'un discours du président Delors devant les représentants des Églises protestantes allemandes, le 14 février 1992. Cf. Archives de la Cellule de Prospective de la Commission européenne, Dossier Marc Luyckx, n51, "Rencontres avec le Président Delors, 1990-1994 ". 
d'un comité de quatre membres chargé de suivre les questions européennes, en coordination avec le ministère grec de l'Éducation, également chargé des cultes. Cependant, il faut attendre septembre 1998 pour que l'Église de Grèce exprime son souhait d'ouvrir un bureau à Bruxelles, animé par un laïc. Cette brusque prise de conscience européenne peut s'expliquer par la volonté d'affirmer un leadership dans la représentation orthodoxe auprès de institutions de l'UE. Celui-ci était justifié par le fait que la Grèce était le seul pays majoritairement orthodoxe de l'Union. Or, en avril de cette année-là s'ouvraient les négociations d'adhésion avec dix nouveaux pays, dont Chypre, majoritairement orthodoxe. L'Église de Grèce se trouvait aussi en situation de concurrence alors que, pour la première fois, un délégué de l'Église orthodoxe de Russie rendait visite à de hauts fonctionnaires de la Commission européenne (avril 1998). Toujours dans une stratégie réactive, l'Église de Grèce nomma, en 2000, à la tête de son bureau bruxellois, un clerc de rang épiscopal, Monseigneur Athanassios d'Achaïe. Cette nomination intervenait au moment de la Convention sur la Charte des Droits fondamentaux, suivie et discutée par les représentations des Églises catholiques et protestantes à Bruxelles, ainsi que par la Fédération humaniste européenne. Finalement, ce n'est qu'en mai 2003 que furent définis les statuts du bureau de l'Église de Grèce à Bruxelles. Monseigneur Christodoulos, archevêque d'Athènes, inaugura cette officine lors de sa visite aux institutions européennes, les 4-6 octobre 2003, c'est-à-dire juste au moment où commençait la CIG qui devait conduire à la signature du Traité constitutionnel par les chefs d'État et de gouvernement (4 octobre 2003).

L'inscription de l'Église orthodoxe de Russie à Bruxelles témoigne d'une forte implication et se situe en rivalité directe avec le Patriarcat œcuménique. En effet, l'Église russe justifia son arrivée à Bruxelles par sa volonté de représenter non seulement l'orthodoxie russe mais aussi la diaspora russe en Europe occidentale et les Églises orthodoxes rattachées au Patriarcat de Moscou (en Ukraine, Biélorussie, Moldavie et Estonie), ainsi que "d'autres pays de l'espace post-soviétique " et les paroisses rattachées au Patriarcat de Moscou sur le territoire de l'Union, ce qui témoigne d'une vision très extensive de son territoire canonique et la pose en rivale du Patriarcat œcuménique. L'ouverture d'un bureau à Bruxelles s'est faite avec prudence et par étapes, en lien avec la représentation permanente de la Fédération de Russie auprès de l'UE. Des dirigeants de l'Église de Russie, de plus en plus haut placés, établirent des contacts successifs avec de hauts fonctionnaires européens afin de jauger la marge de manœuvre de leur institution. En avril 1998, l'archiprêtre Victor Petlyuchenko, vice-président des relations extérieures du Patriarcat de Moscou rendit visite à des officiels européens afin de discuter de la meilleure manière de représenter l'Église de Russie auprès de l'UE. Différentes formules furent testées. En janvier 1999, le métropolite Kirill de Smolensk et de Kaliningrad, 
chef du Département des relations extérieures et numéro deux du Patriarcat de Moscou, effectua un voyage officiel à Bruxelles, à la suite duquel le recteur de la cathédrale orthodoxe Saint Nicolas de Bruxelles, l'archiprêtre Nedossekine, fut invité aux réunions de briefing organisées par la Cellule de Prospective ${ }^{11}$ de la Commission pour les représentants religieux et humanistes (première invitation le 17 décembre 1999). Le 17 juillet 2002, le Saint Synode de l'Église orthodoxe russe décida de créer une " Représentation permanente de l'Église orthodoxe de Russie auprès des institutions européennes ". Celle-ci commença son travail en octobre 2002. À sa tête fut nommé Monseigneur Hilarion de Podolsk, ancien secrétaire aux relations inter-chrétiennes du Département des relations extérieures du Patriarcat œcuménique.

Très vite, l'Église orthodoxe de Russie a fait montre d'une forte implication avec un bureau étoffé de quatre permanents (contre deux pour l'Église orthodoxe de Grèce et quatre pour le Patriarcat œcuménique). Depuis novembre 2002, elle publie sur son site Internet un bimensuel trilingue (anglais, français, allemand), Europaica. Par comparaison, la COMECE édite un mensuel sur papier en cinq langues et la KEK, un trimestriel trilingue sur papier. À la manière des organisations chrétiennes présentes à Bruxelles qui organisent séparément ou conjointement des conférences, l'Église orthodoxe de Russie propose, depuis la rentrée 2004, une conférence mensuelle. Alors que, pour les protestants et les catholiques, ces réunions visent autant à mobiliser leur propre réseau qu'à sensibiliser les fonctionnaires ou parlementaires européens à leur problématique, l'Église russe a plutôt tendance, à travers ces réunions, à mettre en scène son poids géopolitique en Europe en invitant, par exemple, des représentants des Églises orthodoxes de l'Union européenne. Elle démontre ainsi sa sphère d'influence en Europe occidentale tout en servant de recours aux Églises orthodoxes qui cherchent à prendre contact avec les institutions européennes. L'Église russe, avec la collaboration du diocèse de Bruxelles et de Belgique (Patriarcat de Moscou) et de la Fraternité orthodoxe de Belgique, a

\footnotetext{
${ }^{11}$ La Cellule de Prospective est un " think tank " rattaché à la Présidence de la Commission. Il conseille le président tout en menant des études de moyen/long terme. C'est à ce niveau politique et transversal qu'ont été organisées les relations Commission/religions à partir de Jacques Delors. Sous la présidence de Romano Prodi, la Cellule fut remplacée par le Groupe des Conseillers politiques (GOPA) et perdit beaucoup de son caractère prospectif. L'un des quatre conseillers principaux était chargé du suivi des relations avec les religions et les humanistes. Le Président Barroso semble avoir reconduit la formule prodienne plutôt que deloriste. Pour une analyse plus détaillée des relations Commission/religions, voir Massignon (Bérengère), op. cit., chapitre II : " Le "laboratoire" des relations Commission européenne/religions : Définition de référentiels d'action et forums institutionnalisés "; Massignon (Bérengère), "Les relations entre les organismes religieux européens et les institutions de l'Union européenne : un laboratoire de gestion de la diversité religieuse et philosophique ? ", in Armogathe (Jean-Robert), Willaime (Jean-Paul), éds., Les mutations contemporaines du religieux, Turnhout : Brepols, 2003. Voir aussi la contribution de deux acteurs clés de ces relations, l'un religieux, l'autre haut fonctionnaire européen : Charentenay (Pierre de), "Les relations entre l'Union européenne et les religions ", Revue du Marché Commun et de l'Union européenne, (465), février 2003 ; Jansen (Thomas), " Europe and Religion : The Dialogue between the European Commission and the Churches or Religious Communities n, Social Compass, 47 (1), 2000.
} 
ainsi inauguré son cycle de conférences par l'invitation du métropolite Jérémie de Wroclaw, membre du synode de l'Église orthodoxe de Pologne (13 octobre 2004) et de Monseigneur Christophore, archevêque de Prague, un des quatre hiérarques de l'Église orthodoxe autocéphale des territoires tchèques et slovaques (11 novembre 2004).

\section{Des visions de l'orthodoxie en Europe : le clivage entre Patriarcat oecuménique et Églises nationales}

La concurrence des représentations orthodoxes à Bruxelles ne repose pas seulement sur des ambitions géopolitiques rivales ${ }^{12}$. Elle tient aussi à des visions différentes de l'Europe et diverses manières d'être présentes auprès des institutions européennes. À ces deux niveaux apparait un clivage net entre Patriarcat œcuménique et Églises nationales.

Le Patriarcat œcuménique représente avant tout une autorité spirituelle et morale alors que les représentations des Églises orthodoxes nationales à Bruxelles cherchent à peser sur les développements de la construction européenne par les divers moyens du lobbying. Symptomatiquement, l'Église orthodoxe de Grèce et celle de Russie ont déposé des contributions à la Convention pour la définition d'un traité constitutionnel alors que le Patriarcat œcuménique ne l'a pas fait. Comme les Églises nationales protestantes, notamment l'Église évangélique d'Allemagne (EKD), très implantée à Bruxelles, mais aussi l'Église catholique (même si cela ne s'exprime pas par des voies nationales), l'Église orthodoxe de Grèce cherche à se prémunir des retombées que la législation communautaire pourrait avoir sur sa position privilégiée, notamment par le biais de l'application du principe de non-discrimination. Le même raisonnement vaut pour l'Église de Russie dans ses rapports avec le Conseil de l'Europe dont la Russie est membre ${ }^{13}$. Il s'agit d'un lobbying avant tout défensif. Extérieure au territoire de l'Union, l'Église orthodoxe russe partage aussi des caractéristiques avec le lobbying des organisations de la communauté juive, majoritairement implantée hors d'Europe. Il s'agit pour elle de défendre la diaspora russophone en Europe occidentale et de favoriser de bonnes relations entre l'Union et la Fédération de Russie, comme le Congrès

\footnotetext{
${ }^{12}$ Sur la géopolitique de l'orthodoxie, voir notamment : Bardos-Feltoronyi (Nicolas), Églises et États au centre de l'Europe. Réflexions géopolitiques, Paris : L'Harmattan, 2000 ; Thual (François), Le douaire de Byzance, territoires et identités de l'orthodoxie, Paris : Ellipses, 1998 ; Zinovieff (Maurice), L'Europe orthodoxe, Paris : Publisud, 1994.

${ }^{13}$ L'Église orthodoxe russe a non seulement un bureau à Bruxelles, mais aussi un à Strasbourg chargé de suivre les travaux du Conseil de l'Europe. Elle a vivement réagi aux résolutions de l'Assemblée parlementaire du Conseil de l'Europe des 3-8 octobre 2005 prônant une approche comparative et historique de l'enseignement des religions (résolution 1720/2005) et liant la question des discriminations faites aux femmes à la persistance de valeurs traditionnelles inspirées des religions (résolution 1464/2005).
} 
juif européen défend les intérêts de la diaspora juive d'Europe, tout en voulant favoriser de bonnes relations entre l'Union et Israël ${ }^{14}$. En revanche le Patriarcat œcuménique s'investit dans la promotion de rencontres interreligieuses ou l'organisation de réunions sur des thèmes d'intérêt général comme l'écologie.

Le Patriarcat œcuménique et les Églises orthodoxes nationales ont une vision différente du rapport de l'orthodoxie à l'Europe et au reste du monde. Le Patriarcat de Constantinople a, rappelons-le, un rôle prépondérant parmi les Patriarcats dans la conduite des affaires interreligieuses ${ }^{15}$. Il se distingue par un refus du clash des civilisations, en accord avec les référentiels défendus par les institutions européennes. Il a ainsi joué une activité de médiation dans le conflit yougoslave. Il a, par exemple, organisé, en 2000, à Thessalonique, une conférence avec le soutien de l'OTAN, de la Commission et du Parlement européens. Celle-ci rassemblait une quarantaine de représentants religieux ${ }^{16}$ sur le thème The Contribution of Religious Communities towards Multi-ethnic and Democratic Societies. De même, suite aux attentats du 11 septembre 2001, le Patriarcat œcuménique a organisé, avec le soutien du président de la Commission, Romano Prodi, une vaste rencontre interreligieuse rassemblant une cinquantaine de personnalités religieuses, autour du thème The Peace of God in the World. Towards Coexistence between Monotheistic Religions (19-20 décembre 2001).

A contrario, l'Église orthodoxe de Grèce peut se montrer assez hostile à l'islam. Lors de son discours devant les parlementaires européens, Monseigneur Christodoulos d'Athènes a mis en garde, dans des termes très virulents, contre la possible entrée de la Turquie dans l'Europe ${ }^{17}$. De même, au

\footnotetext{
14 Conférence au Parlement européen, les 3-4 décembre 2004 : " La population russophone dans l'Europe élargie ", Europaica, (54), 21 décembre 2004.

15 Sur les fonctions du Patriarcat œcuménique, voir note 9.

${ }^{16}$ Étaient notamment présents des représentants de la KEK, de la COMECE, du Patriarcat de Serbie et des dirigeants musulmans de Bosnie.
}

${ }^{17}$ Discours du 8 octobre 2003. "The Church demands that the Union remains European, an expression of European Civilization. For a number of centuries we, Europeans, have agreed that Europe is not merely a geographic term, but a cultural identity, from which derives geopolitical importance. If we do not take this realization into account then we are not creating Europe but a new entity, alien and possibly hostile to Europe and its civilization... A Union that would include countries of the Eastern and Southern Mediterranean will constitute a historical joke. Who among us desires a political entity comparable to the erstwhile Soviet Union ? Who among us did not see in the collapse something more than the fall of Communism? Who did not see in it the dissolution of "union" of dissimilar cultures ? The Churches would have had nothing to fear if a policy aimed to incorporate alien cultures was successful. All European Churches are concerned that such policy would have devastating consequences for the European Spirit. Such a policy means that such monstrous "union" would succeed only to the degree that it is able to eliminate ethnic and national identities. Such policy leads to the draining of the European spirit and using its corpse for the construction of a grey mass with the incoherent designation of a "multicultural union". If the European political leadership in fact desires this; why doesn't it have the courage to admit it and why does it persist in calling this union "European" ? ". Cité dans Europaica, (26), 3 novembre 2003. 
rebours de la thématique du dialogue des civilisations, chère aux institutions européennes, de nombreux discours de représentants de l'Église orthodoxe russe, notamment Monseigneur Hilarion en charge de la représentation permanente de son Église auprès de l'UE, présente le rapport orthodoxie/Europe occidentale comme le choc de deux civilisations irréductibles ${ }^{18}$.

Autre différence, le Patriarcat œcuménique se projette dans une perspective européenne transnationale qui est celle des diasporas sur lesquelles il a autorité ${ }^{19}$. Sa vision paneuropéenne n'est d'ailleurs pas strictement inter-orthodoxe, mais avant tout œcuménique et interreligieuse. Il raisonne en terme d'eurorégions multiethniques et multiconfessionnelles. C'est ce dont témoignent la conférence et les manifestations itinérantes de juin 1999 qu'il a organisées sur le thème $A$ River of life : From the Danube to the Black Sea. Ces initiatives concernant la sensibilisation aux problèmes écologiques dans le bassin du Danube, ont rempli les critères européens pour recevoir un financement du programme PHARE, un programme qui finance des rencontres et des collaborations transfrontalières en Europe centrale et orientale. À la différence, les Églises orthodoxes autocéphales sont liées au fait national, jusqu'à parfois défendre une vision ethnique de la nation (le phylétisme) ${ }^{20}$. Quand cela ne les conduit pas à mettre explicitement en adéquation identité nationale et identité religieuse orthodoxe, ces Églises défendent une Europe des patries, voire même une Europe des peuples.

\footnotetext{
${ }^{18}$ De très nombreux discours émanant de Monseigneur Hilarion, responsable de la Représentation permanente de l'Église orthodoxe russe à Bruxelles, développent, de différentes manières, la thématique du choc entre les visions du monde libéral occidental et des orthodoxes ou des sociétés traditionnelles inspirees par des valeurs religieuses. Cette approche fut notamment illustrée dans le discours prononcé à l'occasion du congrès " L'Europe en dialogue. Être chrétien dans une Europe pluraliste ", Gniezno, 16-17 septembre 2005, qui rassemblait des responsables politiques européens et nationaux et des représentants religieux, lors de la rencontre entre le président de la Commission et des leaders religieux à Bruxelles, le 12 juillet 2005, ou encore à l'occasion de la rencontre des associations et des organisations religieuses au Parlement européen, le 22 avril 2005. Ce fut également la perspective adoptée lors de la $8^{\text {ème }}$ rencontre orthodoxie/PPE, à Thessalonique. Celle-ci portait paradoxalement sur le thème Building Europe through Reconciliation and Cooperation.
}

19 Daldas (Nikolaos), " Le statut de la diaspora orthodoxe ", Istina, 1995.

${ }^{20}$ Le phylétisme a été condamné par le Synode de l'Église orthodoxe en 1872 , dans un contexte de constitution d'entités nationales et d'Églises autocéphales en Europe orientale et balkanique. La Patriarcat œcuménique a réitéré la condamnation du phylétisme lors de la Conférence préconciliaire de Chambéry (1996) et lors de la rencontre panorthodoxe de Patmos, en 1995. 


\section{UNE DYNAMIQUE D'EUROPÉANISATION}

Cependant, peser sur le processus de décision européen nécessite des stratégies d'alliance. Les trois représentations orthodoxes ont ainsi développé des stratégies de collaboration intra-orthodoxe, tout en cherchant à tisser des liens avec les structures européennes catholiques et protestantes. En outre, devenir un acteur légitime aux yeux des institutions européennes suppose d'en épouser les thématiques ou du moins de ne pas les heurter de front. L'Église orthodoxe russe a montré dans cette logique une capacité d'adaptation particulière. Elle a pu manier le discours ethnocentrique, traditionaliste et anti-occidental et en même temps adopter une perspective plus nuancée qui lui a permis de négocier sa différence dans le champ européen. Plus généralement, les stratégies d'européanisation de l'orthodoxie ont illustré ce souci de marquer une différence et de lui donner un sens dans le concert européen.

\section{Coopération intra-orthodoxe et réaménagement du jeu œcuménique ${ }^{21}$}

Nouveaux venus sur le terrain des institutions européennes, les représentations orthodoxes ont d'abord cherché à s'intégrer dans le concert des relations œcuméniques bruxelloises. Catholiques et protestants ont, en effet, tissé de longue date des relations à différents niveaux. Si bien qu'on peut parler d'un œcuménisme au quotidien, pragmatique, ordonné à des fins du lobbying. On peut rappeler à ce sujet l'organisation de conférences conjointes auprès des fonctionnaires et parlementaires européens ou les réunions ad hoc des commissions juridiques de la Commission "Églises et Société " de la KEK et de la COMECE pour coordonner leur action de lobbying, etc.

Les représentations orthodoxes à Bruxelles se sont d'abord appuyées sur les liens anciens avec les protestants tissés au sein d'organisations œcuméniques communes. Le Patriarcat œcuménique et l'Église de Grèce, à leur début à Bruxelles, ont demandé à être invités comme observateurs aux réunions de Commission œcuménique européenne pour les Églises et la société (European Ecumenical Commission for Church and Society - EECCS), la structure protestante au niveau de l'Union européenne. Devant le Parlement européen, en

\footnotetext{
${ }^{21}$ Sur les recompositions du champ œcuménique après la chute du communisme et plus particulièrement au niveau européen, voir Bizeul (Yves), " Les stratégies œcuméniques dans un contexte de globalisation ", in Bastian (Jean-Pierre), Champion (Françoise), Rousselet (Kathy), éds., op.cit. ; Willaime (JeanPaul), " L'ambivalence œcuménique de Jean-Paul II. Entre la restauration catholique et la promotion du dialogue n, in Luneau (René), Michel (Patrick), éds., Tous les chemins ne mènent plus à Rome. Les mutations actuelles du catholicisme, Paris : Albin Michel, 1995 ; Willaime (Jean-Paul), "Les formes de coopération des organisations et acteurs religieux en Europe entre œcuménismes et quête identitaire ", in Commissariat au Plan, Institut Universitaire de Florence, Chaire Jean Monnet d'Etudes européennes, op.cit.
} 
1994, le patriarche œcuménique Bartholomé $1^{\mathrm{er}}$ a souligné l'orientation œcuménique ancienne du Patriarcat de Constantinople et son implication dans la création du Conseil œcuménique des Églises (COE), structure mondiale qui rassemble protestants et orthodoxes. Les statuts du bureau bruxellois du Patriarcat œcuménique (1997) mentionnent la collaboration avec le COE, la $\mathrm{KEK}$, la COMECE et avec des ONG chrétiennes à caractère catholique, protestant ou œcuménique. Entre 1980 et 1988, l'Église de Grèce a d'abord envisagé sa présence à Bruxelles à travers un rassemblement inter-chrétien, avant de se doter elle-même d'un bureau ${ }^{22}$.

Cependant, les Églises orthodoxes n'ont pas inscrit durablement leurs stratégies de lobbying européen dans le cadre d'une coopération œcuménique avec les protestants. La volonté de se doter de leurs propres structures peut même être interprétée comme une conséquence de la crise des relations œcuméniques protestants-orthodoxes, due au choc de différentes visions ecclésiologiques et anthropologiques, alors même que le mode de décision au sein des instances internationales et paneuropéennes œcuméniques (Conseil œcuménique des Églises ou Conférence des Églises européennes) favorise les Églises protestantes plus nombreuses ${ }^{23}$.

Par contre, il existe une certaine convergence entre catholiques et orthodoxes sur une réponse traditionnelle à apporter aux questions de société et de mœurs. De plus, la vision de Jean-Paul II d'une " Europe à deux poumons " intègre l'orthodoxie dans l'Europe et fait écho aux discours orthodoxes sur la " maison commune européenne ", terme employé par Mikhaill Gorbatchev au moment de la Perestroïka. Dans son rapport d'activité de 1997, la COMECE se fixe comme priorité le rapprochement avec les orthodoxes. Monseigneur Homeyer, président de la COMECE, effectue plusieurs voyages en Biélorussie (janvier 1999), Grèce et Roumanie (janvier 2000). Ces contacts débouchent sur des invitations à Bruxelles, occasions de premiers contacts avec les institutions européennes. Le métropolite Philaret de Minsk se rend dans la capitale belge en septembre 2000, suivi en décembre de la même année par une petite délégation d'évêques orthodoxes grecs. Sur plusieurs dossiers européens, catholiques et orthodoxes sont sur la même longueur d'ondes. Ils furent d'accord pour supprimer l'initiative " Une âme pour l'Europe ", programme qui finan-

22 Lettre officielle de l'archevêque d'Athènes Séraphin, le 27 février 1979, citée dans Papathomas (Grigorios), L'Église de Grèce dans l'Europe unie. Approche nomocanonique, Athènes : Editions Pektasis, 1998.

${ }^{23} \mathrm{Il}$ y a eu, cependant, quelques rencontres entre protestants et orthodoxes de la KEK à Bruxelles. Ces derniers ont été invités au CES de la KEK pour discuter du document " Les Églises européennes qui vivent leur foi dans la mondialisation ". Ce texte est l'apport du CES à la préparation de la prochaine réunion plénière du COE (8-9 décembre 2005). Il renvoie dos à dos le libéralisme économique et l'économie centralisée totalitaire. 
çait des rencontres interreligieuses sur le sens de la construction européenne ${ }^{24}$, alors que les protestants et les humanistes y étaient plus attachés. Lors de la Convention sur le Traité constitutionnel, catholiques et orthodoxes défendirent la mention de Dieu dans le Préambule, alors que la Commission Église et Société de la KEK soutenait la seule mention des " racines chrétiennes de l'Europe" ".

L'élection du Pape Benoît XVI fut saluée par les responsables orthodoxes comme la possibilité d'une coopération renouvelée entre les deux Églises ${ }^{25}$, notamment sur le plan européen. Monseigneur Hilarion a rencontré le nouveau nonce apostolique auprès de l'UE le 4 novembre 2005. Lors de cet entretien, il fut question du témoignage commun que catholiques et orthodoxes pouvaient apporter sur les valeurs chrétiennes dans les sociétés séculières. Lors de la rencontre entre responsables religieux et hommes politiques européens et nationaux (dont Rocco Buttiglione et Helmut Kohl) qui s'est tenue les 16-17 septembre 2005, à Gniezno, Monseigneur Hilarion appelait à une large coopération catholico-orthodoxe ${ }^{26}$ (" traditionnal Christianity " (sic)) afin de " maintenir la doctrine et la morale chrétienne ". Au niveau européen, les deux confessions chrétiennes devaient prendre des positions communes contre le mariage homosexuel, l'IVG, la contraception, l'euthanasie et l'ordination des femmes. Elles devaient s'unir dans le combat contre le libéralisme, le sécularisme et le relativisme. L'alliance catholico-orthodoxe ne s'étend pas aux protestants assimilés au libéralisme moral et dépourvus, à la différence des Églises catholiques

\footnotetext{
${ }^{24}$ Nous ne développerons pas ici les raisons de l'échec de cette initiative lancée par Jacques Delors qui renvoient autant à un désaccord des partenaires religieux et humanistes du Comité de pilotage qu'à des causes internes à la Commission européenne. Sur la genèse, les transformations et le fonctionnement de ce programme voir le chapitre II de la thèse de Massignon (Bérengère), op.cit.

${ }^{25}$ Le comité de coordination catholico-orthodoxe s'est réuni les $13-15$ décembre 2005. À cette réunion ètaient présents deux représentants européens de l'orthodoxie, Monseigneurs Athanassios et Hilarion. La co-présidence du comité est tenue par le Patriarcat œcuménique. Ses réunions avaient été interrompues en 2000 à cause d'un désaccord sur l'uniatisme. Une rencontre plénière est prévue entre les 18 et 25 septembre 2006. (Cf. Europaica (81), 16 décembre 2005). Un autre exemple du rapprochement catholicoorthodoxe est fourni par la rencontre organisée entre le président du Conseil pontifical Justice et Paix et le métropolite Kirill, à Moscou, à l'occasion de la traduction en russe du Compendium de la doctrine sociale de l'Église catholique (29 novembre 2005). Le vice-président du Département des relations extérieures de l'Église orthodoxe russe a alors proposé une collaboration catholico-orthodoxe dans le service social, sur les questions " de paix, d'éthique scientifique, des rapports de sexes et de justice ". (Cf. Europaica, (80), 6 décembre 2005). Le 28 décembre 2005, le métropolite Kirill a rencontré le Secrétaire du Saint-siège pour les relations avec les États, Monseigneur Giovanni Lajalo, en visite à Moscou. Il a souhaité que catholiques et orthodoxes coordonnent leurs actions dans le cadre des institutions européennes (UE, Conseil de l'Europe) (Cf. Europaica, (79), 10 novembre 2005.) Le $1^{\mathrm{er}}$ mai 2005, le métropolite Kirill a rencontré le Pape Benoît XVI. Il a insisté sur la " nécessité pour les deux Églises d'œuvrer ensemble pour la défense des valeurs traditionnelles en Europe $n$. (Cf. Europaica, (66), ${ }^{\mathrm{er}}$ mai 2005.)
}

26 " Can Europe breathe with one lung ? Catholic-Orthodox dialogue today ", Europaica, (74), 22 septembre 2005 . 
et orthodoxes, d'un corpus de doctrine sociale clairement établi, base doctrinale qui permet de fonder une coopération solide ${ }^{27}$.

Cependant, cette alliance n'est que stratégique. Ainsi, le représentant de l'Église orthodoxe russe auprès de l'UE écarte-t-il le projet du cardinal Kasper, président du Conseil pontifical pour la promotion de l'unité des chrétiens, présenté à Bari, en présence de Monseigneur Kirill, dans le cadre du Congrès eucharistique national italien des 22-29 mai 2005, de créer un "synode de réconciliation catholico-orthodoxe ${ }^{28}$. Cette idée soulève des réticences dans la mesure où elle rappelle les conciles où les catholiques avaient " forcé les orthodoxes à adopter l'uniatisme ${ }^{29}$. À la place d'une unité ecclésiale et dogmatique entre les deux confessions chrétiennes, il propose " une Conférence catholico-orthodoxe des évêques au niveau européen ". Ce terme est calqué sur celui de la COMECE. De plus, choisir le niveau des évêques et non des Églises, permet une égalité entre orthodoxes et catholiques. Ces derniers sont ainsi amenés sur le terrain de l'ecclésiologie orthodoxe, plus décentralisée. Dans le même esprit de recherche d'équilibre, les orthodoxes favorisent le niveau européen. Selon Monseigneur Hilarion, à la différence des autres continents, dans cet espace européen le nombre des fidèles des deux confessions est proche.

C'est dire que le duo - fonctionnel plus qu'idéologique - entre catholiques et protestants, à Bruxelles, ne s'est pas transformé en trio ${ }^{30}$. Il est vrai qu'il y a

${ }^{27} \mathrm{Cf}$. Le Compendium de la doctrine sociale de l'Église catholique et les bases de la pensée sociale de l'Église orthodoxe russe, définies lors du Jubilé du Conseil des évêques de l'Église orthodoxe russe en 2000. Monseigneur Hilarion souligne qu'il existe des convergences plus importantes entre le " christianisme traditionnel ", l'islam et le judaïsme, qu'entre ce premier et le " protestantisme libéral ".

${ }^{28}$ Dans cette " ville pont entre l'Orient et l'Occident ", le Cardinal Kasper fixe l'horizon de la réconciliation catholico-orthodoxe à 2098, soit mille ans après le synode des évẽques grecs et latins de Bari de 1098 . Voir l'agence de presse du Vatican en ligne : 〈www.zenit.org〉, 26 mai 2005.

${ }^{29}$ Dans son discours à Gniezno, le 16 septembre 2005, ("Can Europe breathe with one lung ?n [art.cit.]), Monseigneur Hilarion rejette l'idée d'une union catholico-orthodoxe et prône une alliance stratégique. Pour ce, il s'appuie sur le contentieux uniate en faisant une référence explicite au Concile de FerrareFlorence (1439) qui projetait une union catholico-orthodoxe, à la vieille de la prise de Constantinople par les Turcs, puis au Concile de Brest-Litovsk (1596) qui vit le ralliement de l'Église gréco-catholique d'Ukraine à Rome et sa rupture officielle avec le Patriarcat de Moscou. Cette mémoire historique du contentieux uniate est aujourd'hui ravivée par les conflits entre uniates et orthodoxes autour de la restitution des biens spoliés par le régime communiste et autour du projet de l'Église catholique de créer des diocèses sur le territoire canonique orthodoxe. Cette avancée de l'uniatisme vers l'Est a été interprétée par les Églises orthodoxes comme une violation des accords catholico-orthodoxes de Balamand (1993) qui condamnaient l'uniatisme comme méthode d'union entre catholiques et orthodoxes. Pour un long développement sur l'historique des relations catholico-orthodoxes, voir le discours de Monseigneur Hilarion, "Prospects of Orthodox-Catholic Relations ", prononcé le 7 octobre 2002, à l'Université St Thomas (Minnesota) et le 9 octobre 2002, à l'Université catholique d'Amérique (Washington D.C.). Voir <www.orthodoxeurope.org>.

$3^{30}$ Lors de la rencontre religions/UE des 16-18 septembre 2005, Monseigneur Hilarion appelle à une alliance stratégique entre catholiques et orthodoxes, alors que son homologue protestant ne mentionne, à 
eu quelques réunions tripartites de concertation finale dans le cadre de diverses CIG et Conventions ${ }^{31}$, mais l'essentiel de la coordination avait été réalisé, au préalable, entre catholiques et protestants. Jusqu'à présent, les relations catholico-orthodoxes n'ont pas atteint l'intensité des relations catholico-protestantes. Cependant, le rapprochement catholico-orthodoxe achoppe sur la question uniate. Lorsque l'Église gréco-catholique d'Ukraine a souhaité ouvrir un bureau à Bruxelles fin 2003, son représentant fut invité à faire une conférence au siège du COMECE. La collaboration protestants-orthodoxes reste, quand à elle, difficile au sein de la KEK et ne se traduit pas par des engagements communs en direction des institutions européennes, malgré la recherche de compromis institutionnels ${ }^{32}$.

L'adaptation des Églises orthodoxes européennes au jeu communautaire se fait pourtant progressivement au fil des CIG et des Conventions. Leur lobbying qui était d'abord strictement national s'européanise, tout en s'intensifiant.

Lors de la CIG pour le Traité d'Amsterdam, l'Église orthodoxe de Grèce fit cavalier seul, en faisant pression sur son seul gouvernement. Elle obtint ainsi une déclaration annexe spécifique $\left(n^{\circ} 59\right)$ qui lui permettait de sauvegarder son statut, tel qu'il avait été défini dans le Traité d'adhésion de la Grèce. La déclaration annexe $n^{\circ} 11$, pour laquelle s'étaient battus les catholiques et protestants, avait, en fait, le même but : maintenir les questions religieuses dans le domaine de compétence nationale afin de sauvegarder la variété des statuts juridiques des Églises dans les États membres, avec leurs privilèges, en cas de concordat ou de système des cultes reconnus. Ce texte avait, cependant, une portée plus large puisqu'il valait pour toutes les Églises, les communautés religieuses et les mouvements philosophiques non confessionnels de tous les États membres.

Lors de la Convention pour la Charte (17 décembre 1999-2 octobre 2000), l'Église orthodoxe de Grèce fut présente dans les débats, quoique de manière limitée. Monseigneur Christodoulos intervint très tard alors que les négociations étaient presque closes. Dans une lettre adressée au Président Jacques

propos de l'œcuménisme, que la Charte œcuménique entre catholiques et protestants. Voir Europaica, (74), 22 septembre 2005 .

${ }^{31}$ Par exemple, la réunion tripartite du 7 avril 1997, à la fin de la CIG pour le Traité d'Amsterdam.

${ }^{32}$ Lors de la Convention sur l'avenir de l'Europe, en mars 2001, il y eut une réunion entre des responsables européens orthodoxes (Patriarcat œcuménique et Église orthodoxe de Grèce) et protestants (CES de la KEK, EKD). Chaque confession chrétienne était représentée à égalité avec deux membres. Les Églises orthodoxes visent à obtenir la même égalité dans le processus de décision des instances oecuméniques (COE et KEK). Ce modèle paritaire a été proposé au sein du COE lors de la réunion de son comité central, les 15-22 février 2005. La décísion se prendrait désormais, non à la majorité des voix, mais par consensus ou accord. À cet effet devrait être créé un "Standing Committee for Consensus and Cooperation " réunissant un nombre égal de représentants protestants et orthodoxes. Cette proposition doit être débattue lors de l'Assemblée plénière du COE, en février 2006. 
Chirac, il critiqua la position française contre la mention de l'héritage religieux dans le Préambule de la Charte des Droits fondamentaux.

Tout au long de la Convention sur le Traité constitutionnel, les Églises orthodoxes de Grèce et de Russie furent actives sur la scène européenne et élargirent leur répertoire d'action, sans pour autant fournir un effort de mobilisation comparable à celui des structures européennes d'Églises protestantes et catholique. Ces deux Églises orthodoxes déposèrent chacune une contribution sur le site de la Convention ${ }^{33}$, alors qu'elles ne l'avaient pas fait lors des débats sur la Charte des Droits fondamentaux. Enfin, il y eut une première coordination inter-orthodoxe entre les trois représentations basées à Bruxelles, le 7 décembre 2002, dans le contexte de la présentation du projet préliminaire du Traité constitutionnel. De son côté, l'Église orthodoxe de Grèce prit l'initiative d'organiser deux réunions inter-orthodoxes paneuropéennes à Héraklion, les 18-19 mars 2003, puis à Athènes, les 4-6 mai $2003^{34}$, soit à l'époque où la Convention discutait de l'article 2 sur les valeurs de l'Union, puis s'opposait sur l'opportunité d'inclure la mention des " racines chrétiennes " dans le Préambule du Traité constitutionnel. Il faut noter, qu'à plus long terme, les réunions organisées par l'Église orthodoxe russe à son siège bruxellois visent aussi à renforcer les liens inter-orthodoxes. Ainsi, Monseigneur Athanassios d'Achaïe, représentant de l'Église orthodoxe de Grèce à Bruxelles, fut invité à y parler le 7 décembre 2004 .

\section{Les dynamiques d'européanisation orthodoxes : des spécificités négociées ?}

Nous avons souligné plus haut la différence des modes de présence et des visions de l'Europe du Patriarcat œcuménique et des Églises orthodoxes nationales. Le clivage n'est pas aussi strict, dans la mesure où les Églises autocéphales négocient leur différence dans l'Europe et développent une rhétorique complexe entre affirmation de leur spécificité et adhésion à la construction européenne. Selon les personnes, les enceintes, le contexte, elles naviguent entre le registre du particularisme et de l'européen, quitte à faire de leurs particularités un signe vivant de la diversité intrinsèque à l'Union européenne dont la devise est, après tout, "Unie dans la diversité ".

\footnotetext{
33 Pour consulter l'ensemble des contributions des organisations de la société civile au débat constitutionnel européen, voir le site : 〈http://europa.eu.int/constitution/futurum/forum_convention/doc_fr.htm〉

34 Cette dernière était intitulée " Valeurs et principes pour l'édification de l'Europe ". Elle rassemblait différents hiérarques orthodoxes : le patriarche Bartholomé de Constantinople, l'archevêque Christodoulos d'Athènes et de toute la Grèce, Monseigneur Hilarion, représentant du Patriarcat de Moscou auprès des Institutions européennes, l'archevêque Anastase de Tirana et d'Albanie, des représentants d'autres Églises orthodoxes autocéphales ; ainsi que des représentants d'autres confessions chrétiennes : le cardinal Etchégaray (Église catholique), l'évêque de Londres Richard Chartres (Église d'Angleterre) et des membres du Parlement européen et du gouvernement de Grèce.
} 
Une première caractéristique des Églises orthodoxes est leur recours au politique dans leurs relations avec les institutions européennes35. Ainsi, les Églises orthodoxes de Grèce et de Russie ont bénéficié de la médiation de leur gouvernement respectif dans l'établissement de contacts avec l'UE. La Grèce, membre de l'Union, a pu mobiliser quelques hauts fonctionnaires européens de nationalité grecque afin de faciliter les relations de l'Église avec la Commission, notamment à propos du contentieux sur la mention de la religion sur la carte d'identité grecque. L'Église orthodoxe de Russie vient de formaliser, le 3 novembre 2005, un accord de coopération avec la Mission permanente de la Fédération de Russie auprès des institutions européennes, en définissant un programme d'actions communes pour $2006^{36}$. Il est vrai que les Églises nationales protestantes s'appuient aussi sur leur gouvernement, tout particulièrement lors des CIG où le processus de décision est inter-gouvernemental. Ainsi, les Églises chrétiennes, notamment l'Église évangélique d'Allemagne, ont pu tout particulièrement compter sur la voix du gouvernement allemand. Helmut Kohl a mis son poids dans la balance pour arracher un compromis sur la Déclaration annexe $n^{\circ} 11$ du Traité d'Amsterdam. Corrélativement, les organisations humanistes et laïques, également ancrées dans la réalité nationale, ont pu compter sur le gouvernement belge à maintes reprises, compensant des moyens de lobbying plus faibles sur le plan européen que les structures européennes des Églises. Mais, pour ces deux types d'organisations, le recours à la voie nationale n'est qu'une option parmi d'autres dans une large palette d'actions déployées au niveau communautaire. Le recours au politique par les Églises orthodoxes est-il seulement le signe d'une immaturité de leurs modes de lobbying en direction de l'UE et donc une manière de compenser leurs faibles moyens de pression sur le plan communautaire? Une stratégie d'européanisation du lobbying orthodoxe semble, peu à peu, se mettre en place, quoique de manière encore très limitée, comparée aux autres confessions chrétiennes et aux organisations représentant l'humanisme séculier.

Il n'en reste pas moins que le recours au politique apparaît, en dernier ressort, comme une caractéristique, sinon une spécificité orthodoxe. Alors que les partis démocrates-chrétiens se déconfessionnalisent ${ }^{37}$ (le Parti populaire européen est plus un grand parti conservateur qu'un parti de la Démocratie chrétienne), des députés des Parlements nationaux issus de différents pays orthodoxes ont cherché à créer un équivalent du parti démocrate-chrétien

\footnotetext{
35 Sur la conception orthodoxe des relations Église/État, voir pour la Grèce : Kokosaloakis (Nikos), “ Orthodoxie grecque, modernité et politique ", in Davie (Grace), Hervieu-Léger (Danièle), éds., Identités religieuses en Europe, Paris : La Découverte, 1996 ; et pour les Balkans : Gillet (Olivier), op.cit.

${ }^{36}$ Europaica, (79), 10 novembre 2005.

37 Lamberts (E.), “ La démocratie chrétienne en Europe comme expression politique des religions chrétiennes : essor et déclin (1945-2000) ", Social Compass, 47 (1), 2000.
} 
européen : la European Inter-Parliamentary Association of Orthodoxy (EIAO,) créée en 1993, à l'initiative de députés grecs ${ }^{3}$. Le pouvoir se partage entre la Grèce (seul membre orthodoxe de l'UE) dont est issu le secrétaire général et la Russie (seul membre permanent du Conseil de Sécurité de l'ONU) à laquelle est dévolue la Présidence. Ces deux pays sont membres permanents du Secrétariat. Cette association s'assigne comme but de " renforcer le rôle de l'orthodoxie dans le cadre de l'Union européenne et de consolider l'orthodoxie comme une entité politique, culturelle et spirituelle, importante et nécessaire, tournée vers la construction d'une nouvelle réalité européenne ". Il s'agit de défendre la place de l'orthodoxie en Europe en rappelant que l'Europe ne s'arrête pas à sa partie occidentale, d'affirmer une solidarité envers les "orthodoxes persécutés " et de s'unir pour " combattre la désinformation qui consiste à imputer aux populations orthodoxes un rôle dans les régions en conflits "39. La question du prosélytisme et des sectes qui cherchent "à changer la carte religieuse ou culturelle de l'Europe " est un sujet de préoccupation de l'EIAO, notamment de sa commission d'experts sur l'éducation $4^{\circ}$.

À l'instar de l'EIAO, qui cherche à traduire sur le plan politique une alliance inter-orthodoxe transnationale, l'Église orthodoxe de Russie a soutenu le projet d'une réunion visant favoriser l'émergence d'un parti orthodoxe en Europe, protecteur des minorités russophones. Les 3-4 décembre 2004, la conférence sur la population russophone d'Europe, organisée au Parlement européen en présence d'une députée européenne russe (Lettonie) ${ }^{41}$, se donnait pour but de créer un parti européen de la population russophone, parti commun à la diaspora russe dans l'UE (" le Parti russe de l'Europe "). Ce parti s'as-

$3^{8}$ Elle comprend, au départ, des députés des pays suivant : Arménie, Bulgarie, Géorgie, Grèce, Estonie, Chypre, Lettonie, Belarus, Lituanie, Moldavie, Serbie-et-Monténégro, Ukraine, Fédération de Russie, Finlande. Puis vinrent s'adjoindre l'Albanie et le Kazakhstan. Depuis 200o, le mouvement s'est internationalisé avec des délégués invités issus de Palestine, Ouganda, États-Unis et Australie ainsi que des représentants issus de pays de l'UE (Pays-Bas, Suède).

39 Acte fondateur et Manifeste de l'EIAO, Voir 〈www.eiao.org〉.

$4^{\circ}$ Déclaration de Chalkidiki réitérée lors de la $5^{\text {ème }}$ session de l'EIAO (1999) qui demande " de renforcer les moyens légaux en faveur des religions traditionnelles locales par l'éducation, l'ècole, l'accès des religieux aux institutions (armée, hôpitaux, prisons), la présence dans les médias et notamment à la télévision; de permettre les mesures législatives et administratives permettant de protéger les victimes des activités religieuses exerçant une pression violente ou inadéquate sur les personnes en détresse ou dans le besoin ", Bulletin de l'ELAO, février 2000, pp. 23-24. La réunion du groupe éducation demande à l'État d'assurer la protection des citoyens et tout particulièrement des jeunes contre de telles pressions (mai 2002). Le même rejet du prosélytisme est opéré au nom du Traité de Rome (sic!), dans la déclaration de l'Église orthodoxe de Grèce sur l'avenir de l'Europe, du 30 mai 2002.

${ }^{41}$ Le comité d'organisation de cette conférence réunissait une députée européenne russe (Lettonie), le président d'une association de la diaspora russe, le Conseil international des Russes, le secrétaire du Conseil de coordination pour les Droits de l'Homme en Lettonie et le secrétaire de la Représentation de l'Église orthodoxe russe auprès des institutions européennes. La conférence se tint au Parlement européen, avec le soutien du parti Verts/EAC dont était membre la députée lettone. Ont pris part à la réunion des représentants russophones de plus de dix pays de l'Union européenne. 
signait comme but : la sauvegarde de l'identité culturelle de la diaspora russophone dans l'Europe élargie et la défense de ses intérêts spécifiques, l'amélioration des relations UE/Russie et l'étude de questions d'intérêt général européen, agenda qui rappelle celui de maintes organisations communautaires juives européennes. Les différents messages émanant de l'Église orthodoxe de Russie (notamment du patriarche Alexis II et du métropolite Kirill) soulignaient le rôle de l'Église dans la préservation de l'identité russe de la diaspora russophone d'Europe. Elle affirmait ainsi son rôle géopolitique en Europe occidentale par le biais de la diaspora russophone, qui pourtant échappe parfois à son contrôle.

Les Églises orthodoxes cherchent à donner sens à leur différence dans le concert européen. Pour elles, leur ecclésiologie collégiale est un modèle en affinité avec la structure confédérale de l'Europe au rebours du centralisme monarchique romain. Comme chez les protestants, la crainte de "l'Europe vaticane ", conjugue méfiance à l'égard du modèle ecclésiologique romain et rejet d'une construction européenne qui rimerait avec une uniformisation et un déni des différences culturelles. Dans son discours du 19 avril 1994 devant le Parlement européen, le patriarche Bartholomé $1^{\mathrm{er}}$ fait un parallèle entre le principe communautaire de subsidiarite et la dialectique orthodoxe entre autocéphalie et synodalité.

C'est surprenant que l'organisation vraiment démocratique de l'Église chrétienne orthodoxe - avec son haut degré d'autonomie administrative et d'autorité locale des évêques, des patriarches et des Églises autocéphales, à laquelle s'ajoute en même temps l'unité eucharistique dans la foi - fasse figure de prototype, lequel a été récemment institutionnalisé par l'Union européenne sous la dénomination de principe de subsidiarité, comme étant la méthode la plus profitable quant à l'articulation des pouvoirs. $4^{2}$

De même, il interprète la devise européenne "Unie dans la diversité " à la lumière de sa foi trinitaire : "Que le sens de l'Europe soit la personne qui tâtonne vers l'autre, dans l'écoute, le respect et la responsabilité. Unité diverse, diversité une, à l'image peut-être d'un Absolu qui est lui-même Communion ".

L'Europe prônée par les représentants orthodoxes n'est pas fédérale mais confédérale. Elle doit respecter la diversité des peuples. Lors de la $9^{\text {ème }}$ Assemblée de l'EAIO, le patriarche roumain Teoctist donnait une justification théologique à cette conception politique en évoquant la métaphore de la Pentecôte, marquée par le don des langues. "Votre Assemblée confirme et incarne le 
merveilleux miracle de la Pentecôte, le miracle de la descente de l'Esprit Saint ; unité dans la différence. L'orthodoxie apprécie la valeur culturelle de chaque pays, de chaque langue, de l'art de chaque nation qui représentent toujours, selon la parole des Saints Évangiles, l'identité nationale, historique et culturelle "43. Lors de la Convention sur l'avenir de l'Europe, les Églises orthodoxes de Grèce et de Russie défendaient, dans leur déclaration, la sauvegarde de la diversité culturelle44.

Une première stratégie des Églises orthodoxes nationales consiste à négocier leur spécificité et leurs intérêts européens, en faisant des concessions formelles ou à la marge. Certaines thématiques des Églises orthodoxes nationales peuvent heurter de front les idéaux européens universalistes et libéraux. Dans différentes déclarations, y compris celles faites au moment de la Convention sur le Traité constitutionnel, en 2002, les Églises de Russie et de Grèce présentent la liberté religieuse, en termes de droits collectifs plus qu'individuels. Cette approche leur permet de justifier un certain protectionnisme religieux et de légitimer la mobilisation contre le prosélytisme des autres religions. À cette fin, ces deux Églises interprètent la Déclaration annexe $n^{\circ} 11$ comme un principe de clôture de leur espace canonique alors que ce texte reconnaît seulement les statuts juridiques des cultes tels qu'ils sont définis, dans leur diversité, au niveau des États membres. Cette déclaration applique le principe de subsidiarité aux questions religieuses (la définition des statuts cultuels revient aux États), mais ne renonce pas à légiférer en matière de Droits fondamentaux (liberté religieuse, principe de non-discrimination, etc.).

Afin d'éviter les heurts frontaux avec d'autres visions du monde, des représentants orthodoxes peuvent adapter leur discours, sans pour autant renoncer à faire passer un message de défense de leurs intérêts particuliers. Par exemple, lorsque l'Église orthodoxe de Russie proclame son rôle de défenseur des diasporas russophones, elle ne se réfère pas à un groupe ethnique (les Russes), mais linguistique (les russophones), voire même à la " la participation à la culture russe ". Ceci lui permet évidemment de définir un périmètre d'action beaucoup plus large que si elle s'adressait aux seuls ressortissants de la Fédération de Russie. Elle peut ainsi englober les diasporas anciennes acculturées à la société occidentale et tous les russophones de l'ancien Empire soviétique 45 .

43 〈www.eiao.org〉.

44 Il en va de même de la position des Jeunes de l'Église orthodoxe de Grèce pour " l'avenir de l'Europe ", présentée à la Convention en grec. Nous remercions Lina Molokotos pour sa traduction.

45 Conférence " la population russophone dans l'UE élargie ", en présence de la seule députée russe de l'UE (Lettonie), Tatiana Zdanoka. Il a été question de créer un parti européen de la population russophone parti commun à la diaspora russe dans l’UE. Cf. Europaica , (54), 21 décembre 2004. 
Une deuxième stratégie est de manier les différents ressorts de la dialectique unité/diversité, particularité orthodoxe/européanité. Prôner "l'unité dans la diversité " est un discours réversible selon que l'on insiste sur l'unité ou l'irréductible diversité. Un même discours devient alors alternativement inclusif ou exclusif. Le discours de l'identité est un discours de la frontière et de l'écart. Or, ces deux notions sont relatives, tout particulièrement dans le contexte post-national qui caractérise l'Union européenne (notion de subsidiarité, gouvernance multi-niveaux).

Les Églises orthodoxes ont une vision géopolitique spécifique de l'Europe compte tenu de leur position géographiquement et numériquement marginale dans l'Union européenne. Inclure l'orthodoxie dans l'Europe tout en lui conservant un rapport de forces favorable par rapport aux autres confessions chrétiennes, signifie dépasser la vision de l'Europe des 15 , voire des 25 , au profit de celle d'une grande Europe "de l'Atlantique à l'Oural "46. De nombreux responsables orthodoxes, religieux ou politiques, emploient la métaphore gorbatchévienne de la " maison commune européenne " 47 , complétée et renforcée par l'adjectif " pluraliste ". Le discours peut correspondre à une vision d'unité dans la diversité, mais peut aussi contenir les germes d'un affrontement Est/Ouest, orthodoxie/Occident. L'orthodoxie serait alors un territoire proposant une vision du monde et un modèle socio-économique et politique alternatif $4^{8}$. La frontière entre discours inclusif et exclusif est relative : dans le souci de représenter une alternative culturelle et spirituelle à l'Ouest déchristianisé, l'Église orthodoxe de Russie, dans sa déclaration lors de la Convention pour l'avenir de l'Europe, se pose en protectrice des valeurs des nouveaux entrants slaves face à l'Occident libéral, sécularisé et dépravé. L'image des heurts entre blocs civilisationnels peut céder la place à la métaphore du pont 49 . L'orthodoxie, notamment par le biais de la Russie eurasiatique, est ce pont entre l'Orient et l'Occident du fait de sa situation géopolitique, mais aussi de son identité civilisationnelle.

De même, le discours de certains responsables de l'Église orthodoxe russe sur le clash des civilisations entre l'orthodoxie et l'Europe occidentale, peut devenir une opposition entre les défenseurs d'une identité religieuse et de valeurs traditionnelles, inspirées par la religion, contre l'Ouest libéral, athée, relativiste et amoral. Elle en vient ainsi, paradoxalement, via un discours

${ }^{46}$ Déclaration de Chalkidiki et propos du président du Parlement hellénique, 〈www.eiao.org〉.

47 Rev. Georges Tsetsis, Représentant du Patriarcat oecuménique lors de la $4{ }^{\text {ème }}$ rencontre entre le PPE et le Patriarcat œcumenique, Fundamental Rights from the Perspective of the Church, p. 67.

$4^{8}$ Ce discours se retrouve chez des dirigeants orthodoxes aussi bien politiques (EIAO) que religieux (Église orthodoxe de Russie). Voir leur site Internet respectif, 〈www.eiao.org〉 et 〈www.orthodoxeurope.org>.

49 Déclaration de Chalkidiki, 〈www.eiao.org〉. 
d'opposition aux valeurs libérales, à prôner une union de toutes les religions, y compris le judaïsme et l'islam, voire au-delà. Un discours d'affrontement devient par la même occasion un ferment d'alliances interreligieuses ${ }^{50}$. Ainsi, l'Église orthodoxe de Russie s'assure la légitimité idéologique pour une implication dans les rapports interreligieux, concurrençant sur son terrain le Patriarcat œcuménique ${ }^{51}$.

On peut se demander si la dialectique de l'unité dans la diversité ne permet pas finalement de justifier n'importe quelle différence dans la mesure où elle devient un signe vivant de la pluralité. Ainsi, retournant l'argument du clash des civilisations dû à l'ancrage dans des identités religieuses différentes, Monseigneur Hilarion, en vient à imputer l'existence de l'affrontement à un " totalitarisme libéral " $5^{2}$ - oxymore s'il en est. Il devient alors légitime d'affirmer diverses interprétations des droits de l'Homme, parfois au rebours des principes élémentaires d'égalité et de liberté. Ainsi, le représentant de l'Église orthodoxe russe auprès de l'UE distingue deux niveaux dans les droits humains : un niveau universel renvoyant aux grands interdits communs à toutes les religions (tu ne tueras point...) et un niveau particulier correspondant aux modalités d'application des libertés fondamentales en fonction des systèmes sociopolitiques particuliers. Il serait faux de penser que ce discours est strictement relativiste, renvoyant dos à dos les adversaires. En définitive, le primat du collectif sur l'individuel, prôné par l'orthodoxie, est posé en alternative au modèle occidental individualiste qui conduit à l'anomie.

\section{CONCLUSION}

La présence orthodoxe auprès des institutions européennes est récente. Elle tient à des logiques externes, telles que la perspective de l'élargissement, la crise du mouvement œcuménique réunissant des protestants et des ortho-

$5^{\circ}$ Différentes déclarations de l'Église orthodoxe de Russie suivent ce schéma. Tel est le cas pour la déclaration du Département des Relations extérieures à propos de deux résolutions du Conseil de l'Europe d'octobre 2005 (voir note 13). Pour infléchir l'attitude des organisations internationales, un front commun des religions, est souhaité afin de combattre les " préjugés matérialistes, syncrétiques, agnostiques ou athées " qui sous-tendent la faveur accordée aux cours comparatifs d'histoire des religions par rapport à une instruction religieuse de nature confessionnelle. Cf. Europaica, (81), 16 décembre 2005.

${ }^{51}$ Ainsi, Monseigneur Hilarion a représenté l'Église orthodoxe de Russie à la deuxième réunion de l'Assemblée des responsables religieux du monde (the Board of World Religious Leaders) qui se tenait à Taiwan les 28-29 novembre 2005 (thème de la rencontre : " la crise du sacré ").

${ }^{52}$ L'expression est employée dans la déclaration du Département des Affaires extérieures de l'Église orthodoxe de Russie, suite à deux résolutions, jugées anti-religieuses, du Conseil de l'Europe, en octobre 2005. Voir note 13. Europaica, (81), 16 décembre 2005. 
doxes et le message adressé par Jacques Delors à toutes les religions afin de donner sens au projet européen d'après Maastricht. Les consultations entre la Présidence de la Commission et des responsables religieux induisent une logique de pluralisation de la scène religieuse européenne, sur un mode compétitif. Le succès des uns entraîne le désir des autres d'avoir le même accès aux institutions, ce qui signifie plus une reconnaissance symbolique que des avantages substantiels. Ce raisonnement s'applique à l'orthodoxie dans ses relations aux autres confessions chrétiennes présentes de longue date à Bruxelles ; il joue également dans les rapports qu'entretiennent les diverses représentations orthodoxes, chacune prétendant au leadership. Les raisons de cette présence dans le champ européen sont également internes. Avec la chute du mur de Berlin, les Églises orthodoxes se sont heurtées à une situation nouvelle d'ouverture du marché religieux. Elles ont pris conscience du processus de globalisation, dont la construction européenne est l'un des avatars. D'où la volonté d'être présentes sur le plan continental. La globalisation bouscule des positions acquises mais ouvre aussi des opportunités d'expansion, de diffusion et d'alliances. Comme le souligne Jean-Paul Willaime, l'Europe est " œcuménogène ", mais donne aussi lieu à des réaffirmations identitaires confessionnelles 53 (coopération inter-orthodoxe) qui s'expriment, entre autres, contre d'anciennes alliances (orthodoxes-protestants) et en faveur de nouveaux liens (catholiques-orthodoxes). Le rapport des trois confessions chrétiennes à Bruxelles illustre la reconfiguration du paysage œcuménique intra-chrétien.

L'analyse des stratégies et des discours des responsables orthodoxes à Bruxelles amène à abandonner toute perspective interprétative culturaliste concernant le rapport orthodoxie/modernité. L'orthodoxie à Bruxelles n'est pas un bloc : il y a trois représentions concurrentes dont l'action est faiblement coordonnée. Les différences organisationnelles correspondent à des clivages entre les visions du Patriarcat œcuménique et des Églises orthodoxes nationales quant à leur conception des rapports orthodoxie/Europe/reste du monde. Cependant, ces clivages ne sont pas absolus. Le discours de l'unité dans la diversité est réversible et dosable. Il est plus ou moins inclusif/exclusif. Il légitime alternativement l'affrontement et les alliances stratégiques. Il permet de négocier sa différence, sans pour autant renoncer à la défense de ses intérêts, ni à prôner l'excellence de son modèle civilisationnel face à un Occident confronté à une crise des valeurs. 